\title{
Use of immunosuppressive medications and prevalence of disability among patients with pediatric systemic lupus erythematosus: initial analysis of the CARRAnet registry
}

\author{
Aimee Hersh ${ }^{6 *}$, Mary Beth F Son ${ }^{2}$, Hermine Brunner ${ }^{3}$, Barbara Anne Eberhard ${ }^{4}$, Emily Von Scheven ${ }^{5}$, \\ CARRAnet Investigators ${ }^{1}$
}

From 2011 Pediatric Rheumatology Symposium sponsored by the American College of Rheumatology Miami, FL, USA. 2-5 June 2011

\section{Purpose}

To describe the demographics, disease features and treatment of participants with pediatric systemic lupus erythematosus (pSLE) enrolled to date in the CARRAnet registry.

\section{Methods}

Subjects were included in the CARRAnet registry, a national pediatric rheumatology database, if diagnosed with pSLE at $\leq 16$ years of age and $\leq$ age 21 at enrollment. Demographics, disease features and prior and current treatments were collected, as well as the SLE disease activity index (SLEDAI), Health Related Quality of Life (HRQOL), ACR Functional Class (current and worst ever), Childhood Health Assessment Questionnaire (CHAQ), and Physician Global Assessment of disease activity (PGA). Associations between parameters were assessed using Spearman's coefficient, and linear regression was used to determine predictors of intravenous (IV) cyclophosphamide (CYC) administration.

\section{Results}

Data from 123 participants enrolled at 20 sites were analyzed; 2 sites contributed 43 participants (35\%). Mean age at onset of symptoms was $13 \pm 3$ yrs (range 3.3-19.9 yrs), and mean age at enrollment was $16.3 \mathrm{yrs} \pm 2.9$ (range 7.121.4 yrs). 42 subjects (34\%) were diagnosed at $\leq 12$ yrs of age. The cohort was $34.1 \%$ white, $40.7 \%$ black, $7.3 \%$ Asian, $3.3 \%$ American Indian, and $14.6 \%$ of participants were

${ }^{6}$ University of Utah, Salt Lake City, UT, USA

Full list of author information is available at the end of the article classified as 'other'. 22 participants (17.8\%) were Hispanic. In the prior 6 months, 3.3\% ( $=4)$ had pulmonary involvement, $4.0 \%(\mathrm{n}=5)$ had myocarditis/pericarditis, and $15.4 \%$ $(n=19)$ developed at least 1 of the following: lupus headache, cranial nerve disorder, organic brain syndrome, psychosis and/or seizure. Since diagnosis, 57 participants (46.3\%) underwent renal biopsy, avascular necrosis developed in $10(8.1 \%)$, and thrombosis in 9 (7.3\%). A summary of medications prescribed are listed in Table 1.

CYC use was not associated with gender, age at onset, CHAQ score, or ACR Functional Class; but was associated with renal biopsy ( $\mathrm{p}<.001$ [CI 4.14-30.6]). 56 participants (45.5\%) were classified as ACR Functional Class III or IV at some point in their disease course, while14 (11.4\%) were considered Class III or IV at the time of enrollment. 54 participants (43.9\%) rated their HRQOL as "very good" or "excellent" but physical function was impaired with $\mathrm{CHAQ}>0$ in $48 \%(n=59)$. The median score for PGA and SLEDAI was 2 (range 0-7) and 2 (range 0-38), respectively. There was a significant association between the CHAQ and SLEDAI scores $(\mathrm{rho}=0.19, \mathrm{p}=0.03)$ or the PGA $(\mathrm{rho}=0.26, \mathrm{p}=0.04)$.

\section{Conclusion}

In the initial analysis of pSLE subjects in the CARRAnet registry, most participants required steroids for disease control and the majority were on additional immunosuppressants. Furthermore, disability occurred in nearly half the cohort, indicating improved treatment strategies are needed to treat pSLE.

(c) 2012 Hersh et al; licensee BioMed Central Ltd. This is an Open Access article distributed under the terms of the Creative Commons 
Table 1 Prescribed treatments in the CARRAnet registry

\begin{tabular}{lll}
\hline Medications & Ever prescribed N (\%) & Currently prescribed N (\%) \\
\hline Prednisone & $119(97)$ & $94(79)$ \\
Methylprednisolone (IV) & $66(54)$ & $4(3)$ \\
Hydroxychlorquine & $116(94)$ & $105(85)$ \\
Mycophenolate mofetil & $72(59)$ & $61(50)$ \\
Cyclophosphamide (IV) & $60(49)$ & $10(8)$ \\
Azathioprine & $27(22)$ & $10(8)$ \\
Methotrexate (oral or injectable) & $32(26)$ & $15(12)$ \\
Rituximab & $26(21)$ & $8(7)$ \\
\hline
\end{tabular}

\section{Disclosure}

Aimee Hersh: None; Mary Beth F. Son: None; Hermine Brunner: None; Barbara Anne Eberhard: None; Emily Von Scheven: None; CARRAnet Investigators: None.

\section{Author details}

'CARRA, Stanford, CA, USA. ${ }^{2}$ Children's Hospital Boston, Boston, MA, USA. ${ }^{3}$ Cincinnati Children's Hospital Medical Center, Cincinnati, OH, USA. ${ }^{4}$ Cohen Children's Medical Center, New Hyde Park, NY, USA. ${ }^{5}$ University of CA San Francisco, San Francisco, CA, USA. 'University of Utah, Salt Lake City, UT, USA.

Published: 13 July 2012

doi:10.1186/1546-0096-10-S1-A28

Cite this article as: Hersh et al:: Use of immunosuppressive medications and prevalence of disability among patients with pediatric systemic lupus erythematosus: initial analysis of the CARRAnet registry. Pediatric Rheumatology 2012 10(Suppl 1):A28.

Submit your next manuscript to BioMed Central and take full advantage of:

- Convenient online submission

- Thorough peer review

- No space constraints or color figure charges

- Immediate publication on acceptance

- Inclusion in PubMed, CAS, Scopus and Google Scholar

- Research which is freely available for redistribution

Submit your manuscript at www.biomedcentral.com/submit 